\title{
El contexto de las reformas del sector de la salud
}

\author{
Carlos Vergara ${ }^{1}$
}

RESUMEN En América Latina, las reformas del sector de la salud han ido de la mano con los fenómenos so ciales y económicos de la segunda mitad del siglo XX y han reflejado el concepto particular del "desarrollo" que ha predominado en distintos momentos. El estancamiento económico y el au mento del gasto social, fenómenos propios de los años sesenta, llevaron a la crisis del modelo de desarrollo basado en la "sustitución de importaciones", vigente desde principios de siglo, y die ron paso paulatinamente al modelo de "globalización" que tuvo inicio en los años ochenta. Del anterior modelo se pasó a una reestructuración de los procesos productivos y a políticas de ajuste económico que redundaron, irónicamente, en una agudización de la pobreza en América Latina. Dos han sido las fases de implementación de este nuevo modelo. La primera, denominada de "re forma social" o de "primera generación", partía del concepto de que la pobreza se reduce a una suma de carencias materiales cuya resolución radica en la repartición equitativa de un volumen fijo de bienes que posee la sociedad. Este marco conceptual, completamente desvinculado de toda connotación histórica y de la política económica, condujo a políticas sociales orientadas por en tero a mitigar la pobreza mediante subsidios destinados a los sectores más necesitados.

En la segunda fase del modelo de globalización, iniciada en los años noventa y denominada "de segunda generación" o de "postajuste", entraron en juego nuevas reglas económicas, basa das principalmente en la competencia internacional, la eficiencia productiva y la transparencia del mercado de capitales. Y si en la primera fase el concepto estratégico de la política social se centraba en combatir la pobreza, en la segunda pasó a convertirse en la búsqueda de la equidad, ya no entendida como la distribución pareja de un volumen fijo de bienes de capital, sino como el ofrecimiento sostenido de mayores y mejores oportunidades para todo el mundo. Acostum bradas al proteccionismo del antiguo modelo de desarrollo, hoy en día las sociedades latinoame ricanas perciben la amenaza de un modelo nuevo que no les ofrece ninguna red de protección social. La viabilidad de las políticas de reforma económica y social de las segunda fase, que se ajustan a las exigencias de un mundo "globalizado", depende, entonces, de poder vencer la des confianza de la población y de lograr el respaldo de una mayoría política, social e institucional.

Un "modelo de desarrollo" puede ser entendido como aquella red compleja de relaciones, de carácter global y de largo plazo, que se establece entre una estrategia de crecimiento económico determinada, un esquema de desarrollo

\footnotetext{
1 Secretaría de la Presidencia, Santiago, Chile. Dirección postal: Palacio de La Moneda, Moneda 1160, Santiago, Chile. Correo electrónico: cvergara@ minsegpres.cl
}

social y un régimen de convivencia política. Los modelos de desarrollo, una vez consolidados, tienden a perpetuarse. Cabe recordar, a manera de ejemplo, que en América Latina el modelo de desarrollo de "sustitución de importaciones" duró más de medio siglo (1).

En las postrimerías de los años sesenta las economías latinoamericanas sufrían de insuficiencias cada vez mayores en sus posibilidades de creci- miento. Dichas economías no crecían o, al menos, no lo hacían a la tasa mínima necesaria para satisfacer las demandas de bienestar crecientes de sus poblaciones. El gasto social se expandía y las posibilidades de financiarlo se restringían. Era imposible, por tanto, seguir financiando una política social que ya se mostraba deficitaria desde hacía algún tiempo. Cabe resaltar, sin embargo, que la crisis del modelo no se 
explica por el estrangulamiento del desarrollo social, sino por la crisis propia de la economía: poco crecimiento, alto déficit fiscal, gran endeudamiento, crisis de la balanza de pagos, alta inflación, etc. El factor que catapultó la crisis en América Latina fue la crisis petrolera de 1973 a 1976, puesto que la consiguiente falta de crecimiento de la economía afectó adversamente, por falta de financiamiento, a las políticas de desarrollo social. Se requería, por tanto, un nuevo modelo de desarrollo, y una nueva estrategia de crecimiento económico comenzó a abrirse paso en nuestro continente. Su punta de lanza fue la política de ajuste estructural (1).

\section{LOS AÑOS OCHENTA: LA TRANSICIÓN DEL ANTIGUO AL NUEVO MODELO}

En los años ochenta, el problema de América Latina era muy sencillo: se tornaba necesario ajustar gastos con ingresos $\mathrm{y}$, como las economías no crecían, la única manera de hacer el ajuste era mediante la reducción drástica del gasto. Era así de simple y claro. El ajuste estructural marcó y definió la transición del modelo de sustitución de importaciones al modelo de la globalización.

Los períodos que marcan la transición de las sociedades de un modelo de desarrollo a otro suelen ser particularmente críticos para la población. En este caso, la transición estuvo marcada por una reestructuración de la producción y por un ajuste estructural de la economía. Tuvo, y aún tiene, efectos directos y claros de orden social. Por una parte quebraron las empresas que ya no po- dían competir con los productos extranjeros, con el consiguiente y muy notable aumento del desempleo; por otra, el ajuste estructural significó una brusca caída del gasto social y de los salarios y remuneraciones. El resultado de ambos procesos fue un aumento muy marcado de la pobreza en América Latina, tanto en su extensión (porcentaje de familias pobres) como en su intensidad (grado de pobreza) (cuadro 1) (2).

Claramente en el cuadro 1 se presentan datos agregados, y no todos los países empezaron su reforma económica al mismo tiempo. Sin embargo, si se sitúa el inicio de estos procesos poco más o menos en la década de los ochenta, se puede observar que entre 1980 y 1990 el nivel de pobreza en el continente subió de 35 a $41 \%$ del total de los hogares. Además, en términos absolutos, las personas pobres aumentaron de casi 136 millones en 1980 a algo más de 200 millones en 1990. Cabe agregar que este aumento de la pobreza fue básicamente un fenómeno urbano. De los casi 65 millones de nuevos pobres, 60 millones (92\%) eran de origen urbano. Ello se explica en gran medida por los efectos que tuvieron en el corto plazo las políticas de ajuste estructural y la reestructuración de los procesos de producción (3).

Aumentos tan pronunciados de la pobreza suelen tardar muchos años en encontrar su punto de inflexión. Después de ese primer período de grandes dificultades y de un alto costo social, los países toman una senda de crecimiento económico relativamente acelerado y sostenido. Sin duda alguna, el aumento de la pobreza y del desempleo y la brusca caída del gasto social y de las remuneraciones son productos de las reformas económicas. Quienes idearon y llevaron adelante la reforma económica en América Latina promovieron también, con distintos resultados en cada país, la adopción de un conjunto de programas simultáneos orientados a mitigar la pobreza (4).

\section{LA POLÍTICA SOCIAL DE LA TRANSICIÓN}

La grave crisis de la pobreza correspondió a lo que podría llamarse la primera fase del nuevo modelo de desarrollo (o fase de transición propiamente dicha). Las políticas sociales asociadas con ella — que en momentos distintos se han denominado de "reforma social" o de "primera generación" - tenían por núcleo estratégico el combate contra la pobreza. Estas políticas sociales partían de un concepto de la pobreza que estaba desvinculado de toda connotación histórica, todo proceso social y toda conexión con las políticas económicas. Se entendía la pobreza como la suma de una lista de carencias cuantificables y mensurables que, al aplicárseles determinado índice, daban cuenta del grado de pobreza que sufría una persona o familia $(5,6)$. Tales políticas solían tener dos elementos clave:

- Los subsidios directos y personalizados. Estos subsidios reflejaban una política de "focalización del gasto" que se oponía a las políticas "universalistas" de los años cincuenta y sesenta. Como el gasto social debía reducirse bruscamente, lo importante era conseguir un rendimiento máximo en eficiencia y eficacia.

CUADRO 1. Pobreza e indigencia en América Latina, 1980 a $1990^{a}$

\begin{tabular}{|c|c|c|c|c|c|c|c|c|c|c|c|c|}
\hline \multirow[b]{3}{*}{ Año } & \multicolumn{6}{|c|}{ Población pobre ${ }^{b}$} & \multicolumn{6}{|c|}{ Población indigente } \\
\hline & \multicolumn{2}{|c|}{ Urbana } & \multicolumn{2}{|c|}{ Rural } & \multicolumn{2}{|c|}{ Total } & \multicolumn{2}{|c|}{ Urbana } & \multicolumn{2}{|c|}{ Rural } & \multicolumn{2}{|c|}{ Total } \\
\hline & No. & $\%$ & No. & $\%$ & No. & $\%$ & No. & $\%$ & No. & $\%$ & No. & $\%$ \\
\hline 1980 & 62900 & 25 & 73000 & 54 & 135900 & 35 & 22500 & 9 & 39900 & 28 & 62400 & 15 \\
\hline 1990 & 121700 & 35 & 78500 & 58 & 200200 & 41 & 45000 & 12 & 48400 & 34 & 93400 & 18 \\
\hline
\end{tabular}

Fuente: Referencia 2.

a Las estimaciones corresponden a 19 países de la Región.

b Incluye a la población indigente. 
- Eficiencia y eficacia. Por eficiencia se entendía gastar los pocos recursos disponibles en quienes efectivamente los necesitaban; por eficacia, ejercer un efecto sobre aquellos indicadores que definían el fenómeno de la pobreza (mortalidad infantil, desnutrición, matrícula escolar, empleo, calidad de la vivienda, etc.) $(5,6)$.

\section{LA SOCIEDAD POSTERIOR AL AJUSTE}

Pasados los años más difíciles de la transición de un modelo a otro, cambiaron las reglas de la economía y de la sociedad y se instaló un nuevo modelo de desarrollo: el de la globalización. En lo económico se impusieron la competitividad internacional, la eficiencia productiva y la transparencia del mercado de capitales; en lo social, la flexibilidad laboral y la privatización de los activos. Simultáneamente, el nuevo modelo se vio acompañado de una depresión económica de ciertas áreas geográficas o sectores productivos que no estaban en condiciones de competir en el ámbito internacional; de un sistema educativo deficiente; de una sensación de incertidumbre frente al futuro de los sistemas de pensiones administrados por empresas privadas; y de una falta de equidad en los servicios de salud. Se hizo necesario, entonces, transitar a políticas sociales de segunda generación y ello dio origen a un nuevo interrogante: cómo reorganizar el conjunto de la sociedad en función de esta nueva estrategia de crecimiento económico. Y si en la primera fase del nuevo modelo el concepto estratégico de la política social era la mitigación de la pobreza, en la segunda no era otro que el logro de la equidad (7).

\section{LA EQUIDAD COMO NUEVO EJE}

América Latina constituye la región del mundo con la distribución más desigual de los ingresos y de la riqueza. En general, la instalación del nuevo modelo de desarrollo en su pri- mera fase empeoró aun más la mala distribución. Los países como Chile, que se encuentran en la segunda fase o en lo que podría llamarse el período "postajuste", hasta ahora no han logrado mejorar la distribución y apenas han podido detener su deterioro. Por tanto, el nuevo modelo arrastra el fantasma del aumento de las desigualdades. Si se calcula que la segunda fase del nuevo modelo empezó a partir de los años noventa, se observa un fenómeno central: la pobreza ha tendido a disminuir, pero las desigualdades han tendido a acentuarse (8).

En los años noventa, según cifras de la Comisión Económica para América Latina y el Caribe (CEPAL), de 12 países estudiados solo 4 han mostrado una leve mejoría de la distribución del ingreso; en uno la distribución se ha mantenido igual, y en los otros 7 la distribución del ingreso ha empeorado. Además, en 8 países ha aumentado la participación del 10\% más rico en el ingreso total; en 8 países la razón dada por el ingreso promedio del 10\% más rico (numerador) contra el del $40 \%$ más pobre (denominador) fue superior en 1997 que en 1990; y en 8 países el coeficiente de Gini, que es una medida de la falta de equidad en el ingreso,fue peor en 1997 que en 1990 (2).

$\mathrm{Si}$ a todo lo anterior se agrega que durante la primera fase del nuevo modelo de desarrollo aumentaron notablemente el sufrimiento y la pobreza, resulta evidente que dicho modelo tendrá que legitimarse socialmente entre la ciudadanía y encontrar apoyo en las elites políticas e intelectuales. Todo ello obedece a que el antiguo modelo, basado en la sustitución de importaciones, había construido una red de protección social. La población se sentía protegida contra los riesgos y amenazas propias del ciclo vital: desempleo, enfermedad, vejez, falta de oportunidades, y demás. El Estado, alabado o criticado, era percibido como una garantía eficaz de esta red de protección y el ciudadano se sentía protegido contra el riesgo y la incertidumbre. El concepto del "bien común", cuyo guardián era el Estado, gozaba de buena reputación. Esta red de protección social, esta suerte de "mano invisi- ble" del desarrollo social, se esfumaba con la desaparición del siglo XX (8).

El nuevo modelo de desarrollo no cuenta con una nueva red de protección social, moderna y contemporánea, capaz de constituirse en la base del desarrollo social del nuevo siglo. Desde el punto de vista de los distintos agentes sociales priman sentimientos de desprotección, desconfianza, incertidumbre y vulnerabilidad ante algunas amenazas y riesgos. De hecho, los nuevos equilibrios macroeconómicos se fundaron sobre niveles de gasto social que ya estaban bastante deprimidos (cuadro 2). Las presiones para impedir que subiera el gasto social no eran menores y las cifras sobre el gasto social en América Latina no dejaban mucho margen para el optimismo. Se veían afectadas la salud, la educación, la vivienda, la calidad de vida de la población más desprotegida y vulnerable. Aunque se conocían bien los medios para estimular la economía, se sabía poco sobre la manera de conseguir mayor equidad (8).

La política social no es otra cosa que el proceso de decidir cuánto invertir y en qué para lograr una sociedad más justa, integrada y equitativa. A la hora de idear programas de cooperación y de investigación deberá tomarse en cuenta, por lo tanto, no solo la diversidad de los problemas, sino también la multiplicidad de las posibilidades de inversión(9).

\section{LAS NUEVAS INQUIETUDES}

La viabilidad de la segunda fase del nuevo modelo de desarrollo — la de la sociedad "postajuste" — descansa sobre dos supuestos fundamentales: primero, que es posible definir nuevos umbrales de equidad; segundo, que una mayoría política y social respalda las políticas de reforma económica y social, encaminadas a lograr su buen desenvolvimiento en un mundo globalizado.

El problema estratégico de la reforma social de segunda generación radica en saber conseguir la equidad, lo cual ciertamente no es posible sobre la base de las fórmulas de los años sesenta. La redistribución de la riqueza, 
CUADRO 2. Niveles del gasto público social en América Latina (expresados en cifras promedio en dólares estadounidenses [US\$] y en porcentajes del producto interno bruto [PIB]), 1996-1997a

\begin{tabular}{|c|c|c|c|c|}
\hline Gasto social & $\begin{array}{l}\text { Gasto real per cápita } \\
\text { (US\$ de 1997) }\end{array}$ & Gasto social/PIB & $\begin{array}{c}\text { Gasto social/ } \\
\text { gasto público total }\end{array}$ & $\begin{array}{l}\text { PIB por habitante } \\
\text { (US\$ de 1990) }\end{array}$ \\
\hline Alto y moderadamente alto $\left(\mathrm{CV}^{\mathrm{c}}\right)$ & $975 \quad(0,38)$ & $19,5(0,15)$ & $60,8 \quad(0,16)$ & \\
\hline Argentina & 1570 & 17,9 & 65,1 & 5790 \\
\hline Uruguay & 1371 & 22,5 & 69,8 & 3783 \\
\hline Brasil & 951 & 19,8 & 59,1 & 3214 \\
\hline Chile & 725 & 14,1 & 65,9 & 3557 \\
\hline Panamá & 683 & 21,9 & 39,9 & 2711 \\
\hline Costa Ricad $^{d}$ & 550 & 20,8 & 65,1 & 2030 \\
\hline Moderado (CV) & $353(0,09)$ & $10,5(0,32)$ & $43,4 \quad(0,16)$ & \\
\hline Colombia & 391 & 15,3 & 38,2 & 1442 \\
\hline México & $352^{\mathrm{e}}$ & 7,8 & 52,9 & 3394 \\
\hline Venezuela & 317 & 8,4 & 39,0 & 2681 \\
\hline Bajo (CV) & $109 \quad(0,42)$ & $7,7(0,25)$ & $38,4 \quad(0,17)$ & \\
\hline Perú & 169 & 5,8 & 40,9 & 2139 \\
\hline Paraguay & 148 & 7,9 & 47,1 & 1248 \\
\hline El Salvador & 147 & 7,7 & 26,5 & 1161 \\
\hline Bolivia & 119 & 12,0 & 44,2 & 892 \\
\hline República Dominicana & 107 & 6,0 & 39,0 & 1104 \\
\hline Guatemala & 71 & 4,2 & 42,1 & 964 \\
\hline Honduras & 58 & 7,2 & 31,9 & 722 \\
\hline Nicaragua & 49 & 10,7 & 35,6 & 591 \\
\hline Promedio regional (CV) & $457 \quad(0,99)$ & $12,4 \quad(0,49)$ & $47,2 \quad(0,27)$ & \\
\hline
\end{tabular}

a Los países se presentan en orden decreciente según el nivel de su gasto social durante el período 1996-1997.

b Solo se presentan cifras correspondientes a 1997.

${ }^{c} \mathrm{CV}=$ coeficiente de variación.

d Solo se presentan cifras correspondientes a 1996.

e Esta cifra no comprende el gasto en vivienda. Si este se incluyera, el gasto social real de 1996-1997 oscilaría alrededor de US\$ 446.

cuando esta última se entiende como un volumen fijo de bienes materiales, no contribuye a una mayor equidad y más bien atenta contra las posibilidades mismas de crecimiento e inversión en los plazos mediano y largo. Es necesario, entonces, entender la equidad como una nueva forma de distribuir los flujos del capital a lo largo del tiempo, dejando atrás, a paso lento, la época de los subsidios y entrando de lleno en la de las oportunidades. Lograr la equidad no es otra cosa que ofrecer mayores y mejores oportunidades para todo el mundo, lo cual es posible solamente cuando la economía ha tenido un crecimiento sostenido. Desde el punto de vista del desarrollo social futuro, la cuestión va mucho más allá del volumen total de la inversión en la economía y guarda relación, más bien, con el destino y la distribución de los frutos del crecimiento y de la mayor productividad. En otras pala- bras, la inquietud central es cómo y cuánto debe invertir la sociedad en sí misma y en su propio futuro, en el entendido de que los segmentos más vulnerables deben ser destinatarios de una mayor proporción de la inversión social. Para países con tasas de crecimiento económico altas y sostenidas, el problema radica en determinar el monto y la composición del gasto social o, dicho de otro modo, en reemplazar la noción de "gasto social" por la de "inversión social" para lograr mayores y mejores oportunidades.

\section{EL PROBLEMA \\ DE LAS INSTITUCIONES: LA GOBERNABILIDAD DE LAS POLÍTICAS SOCIALES}

Uno de los obstáculos principales para la implementación de políticas sociales de segunda generación radica en la falta de instituciones que las puedan respaldar. En efecto, muchas de nuestras instituciones públicas derivan de las viejas instituciones del antiguo modelo de desarrollo, herencia del ordenamiento institucional de los años cuarenta y cincuenta, que respondía a objetivos económicos y sociales muy distintos a los que exige la realidad actual. Peor aun, con el tiempo estas viejas instituciones han ido sufriendo modificaciones y reformas parciales que han desfigurado su naturaleza original, muchas veces despojándolas de todo objetivo a excepción del empleo público que generan. La ineficiencia institucional, la duplicación de funciones, la burocracia excesiva y la falta de misión suelen ser características muy frecuentes de las instituciones encargadas de llevar a la práctica las políticas sociales en la actualidad. Pero tener claros los objetivos de una 
política no es suficiente; también es necesario que haya instituciones capaces de cumplirlos. Esto da origen a dos interrogantes, el primero en relación con la eficiencia de la institución, es decir, con la claridad de sus objetivos, su misión concreta y los procedimientos de gestión correspondientes; el segundo, con la compatibilidad entre instituciones con objetivos nacionales y aquellas cuyos objetivos son más bien territoriales. Esto significa, en otras palabras, posicionar en otro nivel el tema de la descentralización. Si bien el centralismo ha sido un rasgo negativo del antiguo modelo, la autonomía completa parece ser una característica no menos negativa del modelo moderno. En este sentido, se impone la necesidad de realizar investigaciones y estudios sobre la manera de generar las condiciones institucionales apropiadas para implantar las nuevas políticas. Del mismo modo, la modernización de los procedimientos propios de la gerencia pública es fundamental para lograr el éxito de las nuevas políticas de desarrollo social (10).

\section{LA HETEROGENEIDAD DE AMÉRICA LATINA}

Sería equivocado referirse a América Latina y el Caribe como si constituyeran un territorio homogéneo donde los conceptos tienen para todos los países la misma valía y el mismo significado. Según se ha señalado, los problemas principales, los conceptos, la arquitectura, y los instrumentos propios de las políticas sociales difieren en las fases primera y segunda del nuevo modelo de desarrollo, como también los niveles del gasto y la inversión social. Pero esta divergencia entre las fases primera y segunda no ocurre de forma abrupta ni radical. Ningún país pasa bruscamente de la fase de transición a la de "postajuste". Se habla de predominios, de matices principales. Sin embargo, es necesario señalar que los países que atraviesan la primera fase, si quieren mirar hacia el futuro con alguna esperanza, deben hacer frente desde ahora a los problemas "de segunda generación" que plantean la equidad y las reformas. Mientras más se globalizan las economías, más deberán los países concentrarse en las políticas de la segunda fase.

En concreto, y a modo de ejemplo, la solución en el largo plazo de los problemas que acarrea la pobreza radica cada vez más en la calidad y la equidad de la educación, y no en los subsidios directos y focalizados. Los países que pasan por la segunda fase enfrentan todavía $-\mathrm{y}$ lo harán por mucho tiempo- situaciones de pobreza extrema que se ven moral y políticamente obligados a abordar. Si se quedan estancados en los problemas de pobreza que se arrastran del pasado, no les será posible responder eficazmente a las exigencias del futuro.

El ingreso per cápita, el gasto social per cápita y la fase en que cada país latinoamericano se encuentra con respecto al nuevo modelo de desarrollo varían, lo cual confiere a nuestro continente heterogeneidad y diversidad. Tener presente esta diversidad en el diseño y la aplicación de las políticas sociales es un imperativo de los nuevos tiempos.

\section{EL PROBLEMA DE LA LEGITIMIDAD}

Como ya se ha señalado, la fase de transición es particularmente difícil para amplios segmentos de la pobla- ción, siendo los jinetes de este apocalipsis el desempleo, la caída del gasto social y la pobreza. En buena parte de América Latina, el antiguo modelo de desarrollo surgió y se desarrolló de la mano con la democracia. Su ocaso y agonía coincidieron con la instalación de gobiernos autoritarios y militares en casi todo el continente americano durante los años ochenta. La sola promesa de un futuro crecimiento de la economía no es suficiente para garantizar la legitimidad de la reforma económica. Esta última y la reforma social deben hoy en día marchar a la par y, con la participación de la población, en un espíritu de democracia y de pleno respeto por los derechos humanos. Ya no es posible aspirar al éxito de las reformas cuando estas se emprenden sin tener en cuenta a los ciudadanos y desconfiando de ellos, vulnerando las bases de la convivencia democrática.

La legitimidad de la nueva estrategia de crecimiento económico está dada por el énfasis en la búsqueda de la equidad social y por el respeto al sistema democrático. $\mathrm{Y}$ hay un aspecto más importante aun: la equidad y la democracia no son meros indicadores de legitimidad, sino que constituyen la condición indispensable para la viabilidad del nuevo modelo.

América Latina tiene ahora la oportunidad de entrar al nuevo milenio por la puerta ancha del progreso, del crecimiento, de la igualdad de oportunidades, del perfeccionamiento de la democracia. Pero como todas las oportunidades, esta también se puede desperdiciar si no se trabaja con voluntad e inteligencia en el momento oportuno. O se entienden las nuevas claves y desafíos del desarrollo social de nuestro continente, o los países de América Latina corren el riesgo de quedarse estancados en tiempos que ya han desaparecido.

\section{REFERENCIAS}

1. Franco R, coord. Pobreza, necesidades básicas y desarrollo. Santiago, Chile: Comisión Económica para América Latina y el Caribe, Instituto Latinoamericano de Planificación Económica y Social y Fondo de las Naciones Unidas para la Infancia; 1982. Santiago, Chile: CEPAL; 1998.
2. Comisión Económica para América Latina y el Caribe. Panorama social de América Latina.

3. Comisión Económica para América Latina y el Caribe, Instituto Latinoamericano de Planificación Económica y Social y Fondo de las
Naciones Unidas para la Infancia. Desarrollo social en los 80. Santiago, Chile: CEPAL, ILPES y UNICEF; 1984.

4. Franco R, Palma E, Vergara C. El impacto social de la crisis: el relanzamiento del desarrollo social. San José, Costa Rica: Cuadernos 
de Ciencias Sociales, Secretaría General FLACSO; marzo de 1989.

5. Vergara C. El impacto de la crisis sobre la educación y los desafíos que provoca. San José, Costa Rica: Oficina del Instituto Latinoamericano de Planificación Económica y Social (ILPES) para Centroamérica y Panamá; abril de 1989.

6. Vergara C. El debate actual sobre las políticas sociales: enfoques y aportes para el momento chileno. En: Debate actual sobre las políticas sociales. Santiago, Chile: Centro de Estudios del Desarrollo; 1991.

7. Programa de las Naciones Unidas para el Desarrollo. Desarrollo humano en Chile, 1998: las paradojas de la modernización. Nueva York: PNUD; 1998.

8. Comisión Económica para América Latina y el Caribe. Transformación productiva con equidad. Santiago, Chile: CEPAL; 1990.
9. Downs C, Solimano G, Vergara C, Zúñiga L. Social policies from the grassroots. San Francisco, London: Westview Press; 1989.

10. Programa de las Naciones Unidas para el Desarrollo. Cooperación política para la gobernabilidad democrática. Nueva York: PNUD; 1996.

ABSTRACT In Latin America, health sector reforms have gone hand in hand with social and economic trends during the latter half of the twentieth century and have reflected the particular concept of "development" that has been in vogue at different times. Economic stagnation and increased social spending, both hallmarks of the 1960s, led to

The context surrounding health sector reforms the decline of the "import substitution" development model, which had prevailed since the beginning of the century, and slowly gave way in the 1980s to the "globalization"model. From the earlier model, a transition took place toward a restructuring of production and a series of economic adjustment policies that led, ironically, to an increase in poverty in Latin America. Implementation of the new model has occurred in two phases. The first, known as the "social reform" or "first generation "phase, sprang from the notion that poverty is the sum of a number of material shortages that can be corrected through an equitable redistribution of a fixed volume of goods belonging to society. This conceptual framework, which was completely devoid of all historical linkages and separated from economic policy, led to social policies whose entire purpose was to mitigate poverty through subsidies targeting the poorest persons in the society.

In the second phase of the globalization model, which arose in the 1990s and became known as the "second generation" or "postadjustment" phase, new economic rules came into play that were based primarily on international competition, efficiency in production, and openness and fairness in the capital markets. And if during the initial stage the conceptual strategy behind all social policy was to fight poverty, in the second stage the strategy became one of achieving equity, which was no longer interpreted as the even distribution of a fixed volume of capital goods, but as the sustained provision of greater and better opportunities for all. Having grown accustomed to the protectionism inherent in the earlier development model, Latin American societies today feel threatened by a new model that offers them no social safety net. The feasibility of economic and social reform policies during the second phase, which reflect the demands of a "globalized" world, thus depends on the ability to overcome people's lack of trust and to garner the support of a political, social, and institutional majority. 